\title{
Optimal Strategies for the Eradication of Asiatic Citrus Canker in Heterogeneous Host Landscapes
}

\author{
S. Parnell, T. R. Gottwald, F. van den Bosch, and C. A. Gilligan
}

First and second author: Centre for Mathematical and Computational Biology, Rothamsted Research, Harpenden, AL5 2JQ, UK; first and third authors: U.S. Department of Agriculture, Agricultural Research Service, Ft. Pierce, FL 34945; and fourth author: University of Cambridge, Department of Plant Science, Downing Street, Cambridge, CB2 3EA.

Accepted for publication 27 July 2009.

\begin{abstract}
Parnell, S., Gottwald, T. R., van den Bosch, F., and Gilligan, C. A. 2009. Optimal strategies for the eradication of Asiatic citrus canker in heterogeneous host landscapes. Phytopathology 99:1370-1376.

The eradication of nonnative plant pathogens is a key challenge in plant disease epidemiology. Asiatic citrus canker is an economically significant disease of citrus caused by the bacterial plant pathogen Xanthomonas citri subsp. citri. The pathogen is a major exotic disease problem in many citrus producing areas of the world including the United States, Brazil, and Australia. Various eradication attempts have been made

economic costs due to the necessary removal of large numbers of host trees. In this paper, a spatially explicit stochastic simulation model of Asiatic citrus canker is introduced that describes an epidemic of the disease in a heterogeneous host landscape. We show that an optimum eradication strategy can be determined that minimizes the adverse costs associated with eradication. In particular, we show how the optimum strategy and its total cost depend on the topological arrangement of the host landscape. We discuss the implications of the results for invading plant disease epidemics in general and for historical and future eradication attempts on Asiatic citrus canker.
\end{abstract} on the disease but have been associated with significant social and
Asiatic citrus canker (ACC) is a bacterial disease of citrus caused by Xanthomonas citri subsp. citri. The disease is a significant exotic disease problem in many hot-humid citrus producing regions of the world (11) and is primarily spread by windblown rain (3). ACC has a diverse host range of citrus and citrus relatives that occur as residential trees in private properties and cultivated trees in commercial plantations (11). Symptoms include fruit blemishing, defoliation, and premature fruit drop with associated reductions in fruit quality and yield (12). Long-standing international quarantines for ACC, combined with diseaseassociated restrictions to export markets, makes the disease a strong candidate for eradication. Eradication involves the elimination of inoculum via the removal of symptomatic trees and their neighbors that are considered to be exposed and therefore possibly presymptomatic or subclinical for ACC (17). However, although past epidemics have been successfully eradicated from Australia, Florida, and South Africa, eradication is costly and not without obstacle (27). A recent eradication attempt in Florida followed the discovery of the disease in a residential tree in Miami in 1995 (27). Over a 10-year period, the eradication program led to the removal of millions of commercial and residential trees at a cost of $>\$ 1$ billion (14) and was subject to numerous court challenges on behalf of residential citrus owners (4). The potential for eradication programs to cause such social and economic conflict highlights the need for optimal eradication strategies which minimize the impact on the healthy host population.

The design of optimal eradication strategies will depend on a variety of epidemiological considerations that encompass the

Corresponding author: S. Parnell; E-mail address: stephen.parnell@ bbsrc.ac.uk

doi:10.1094/PHYTO-99-12-1370

This article is in the public domain and not copyrightable. It may be freely reprinted with customary crediting of the source. The American Phytopathological Society, 2009. complex interaction between the dispersal of the pathogen and spatial heterogeneities in the host landscape. In ecological studies, the spatial distribution of habitat has been shown to be particularly important in the success of invading populations (20,31). For example, studies have shown that certain forms of heterogeneity can increase the rate at which an invading epidemic will spread $(7,23)$. The importance of landscape heterogeneity is also being increasingly acknowledged in the field of plant disease epidemiology $(5,9,10,25,26)$. The ACC host landscape is a complex mosaic of both residential and commercial trees, the specific pattern of which can vary significantly between different citrus producing areas. Empirical studies to test eradication strategies on different host landscapes are clearly not a viable option. Detailed epidemiological models that capture the temporal and spatial dynamics of both epidemic spread and the eradication process are a vital alternative and can be used to quickly assess the outcome of a range of control strategies $(10,18,29)$.

In this paper, we use a spatially explicit stochastic simulation model to demonstrate the effect of different host topologies on the dynamics of an ACC epidemic and eradication program. We focus on two contrasting patterns found in citrus host landscapes, one characteristic of citrus production in Florida and one characteristic of citrus production in Texas. In Florida, residential and agricultural land is often separated with the former predominantly on the coast and the latter predominantly inland, thus resulting in the geographic separation of residential and commercial citrus trees. In contrast, in Texas, clusters of plantations are often interspersed amongst small residential communities leading to the interspersion of residential and commercial trees. In this paper, we study landscapes characterized by either interspersion or separation of the residential and commercial tree populations. Residential and commercial trees constitute considerably different host populations in terms of, for example, the susceptibility and spatial distribution of trees, but are linked through the transmission of inoculum. Therefore, we model these two populations separately and study the interaction of an epidemic between them. 
In particular, we address the following questions. What is the effect of the contrasting host topologies on epidemic progress? Can we identify an optimum eradication strategy which minimizes the impact on the healthy host population? How does the optimum eradication strategy differ for different host topologies?

\section{THEORY AND APPROACHES}

In this section, we first describe the generation of the host distributions, which provide the setting for the epidemic dynamics and the eradication program. We then derive the epidemic simulation model and describe the simulated process of eradication.

Generating the host distributions. To study the effect of the topology of the host distribution on the spatial and temporal dynamics of an ACC epidemic and eradication program, we generate host distributions with different topologies (Fig. 1). Each host distribution consists of host units of either individual residential trees or commercial citrus blocks, the latter of which contain 100 trees per host unit (Fig. 1). The position of each host unit (either an individual residential tree or a block of commercial trees) is allocated via a poisson cluster process (24). Center positions of a predetermined number of clusters of host units of each host type are randomly assigned. Each host unit is randomly allocated a center position of identical type and then positioned randomly within a radial distance of this center point (Fig. 1). Additionally, each commercial block is assigned adjacent to a randomly existing commercial block with probability 0.75 to create irregular contiguous blocks of citrus to replicate the pattern observed in reality where individual commercial blocks seldom occur in isolation. These processes generate a bivariate spatial point pattern of residential host units and commercial host units which represents an artificial host distribution. We consider four artificial distributions consisting of two variations of host density and two variations of host aggregation (Fig. 1). This choice is motivated by the spatial characteristics of the citrus host landscape in the United States where residential and commercial trees are typically found either interspersed with each other in small clusters (as replicated in Fig. 1A and B) or adjacent to each other in distinct regions (as replicated in Fig. 1C and D). The former is indicative of the citrus host landscape in Florida, whereas the latter is more characteristic of Texas. For ease of reference, the host distributions are labeled as high-density interspersed (Fig. 1A), low-density interspersed (Fig. 1B), high-density segregated (Fig. 1C), and low-density segregated (Fig. 1D).

These distributions are designed to be plausible and representative of realistic citrus landscapes but their choice is motivated to provide contrasting results. Density is simulated by restricting the size of the total grid and the radius of each cluster in which hosts are positioned. The density of residential trees in the high-density host distributions (Fig. 1A and C) are based on surveys conducted in areas of continuous housing in southeast Florida in which all citrus trees were identified and densities of approximately $1,000 \mathrm{~m}^{2}$ per tree observed (15). The density of housing units in southeast Florida is known to be among the highest in the United States; therefore, we also considered a lower density of residential trees in the low-density interspersed and low-density segregated (Fig. $1 \mathrm{~B}$ and $\mathrm{D})$. The commercial tree densities were motivated by satellite images of citrus production areas in Florida and Texas. These were accessed using NASA World Wind open source software (http://worldwind.arc.nasa.gov). In U.S. citriculture it is common to plant with approximately $3.5 \mathrm{~m}$ spacing between trees on the same row and $7 \mathrm{~m}$ between trees on different rows. This was confirmed via satellite imagery of citrus plantations in Texas and Florida. This leads to a density of $2,450 \mathrm{~m}^{2}$ per block of 100 trees which we account for during the generation of the commercial host populations to prevent 'overlapping' of blocks. In the high-density interspersed and high-density segregated host distributions (Fig. 1A and C) the within cluster density is approxi- mately $4,000 \mathrm{~m}^{2}$ per block. This is lower than the expected $2,450 \mathrm{~m}^{2}$ that would be observed if blocks were completely contiguous because we account for between-block voids which occur as roads, drainage ditches, and other noncultivatable land types. We also consider lower densities of commercial blocks in the low-density interspersed and low-density segregated (Fig. 1B and D) to account for areas of less intensive citrus production which occur in some areas, particularly where citrus is not the primary crop. The cluster sizes, for both residential and commercial tree types, used in the high-density interspersed and low-density interspersed host distributions were selected to be typical of those found in the Rio Grande Valley citrus producing area of Texas and were informed by satellite images of this area. The total number of clusters modeled was limited by computation time but was chosen to represent a realistic proportion of residential and commercial host units within the grid-size under study.

Simulating the epidemic. The epidemic model is an adaptation of the ACC model introduced by Cook et al. (6). This model describes the spatial and temporal spread of an ACC epidemic in a population of residential citrus trees. We utilize the parameter values from this work which were estimated via a Markov chain Monte Carlo algorithm using spatiotemporal data from an 18month epidemiological study in southeast Florida (15). We extend their one host-type model to account for blocks of commercial citrus trees and consider an epidemic which interacts between the two populations of hosts (individual residential trees and blocks of commercial trees). In the high-density host distributions commercial host units will in some cases be contiguous (Fig. 1A and C). However, even in this case within-block spread of the disease can be modeled independently due to the between-block canopy separation which occurs in citrus plantations as a result of the roads and irrigation ditches that separate blocks. The epidemic is modeled as a discrete time Monte Carlo simulation (1) with the
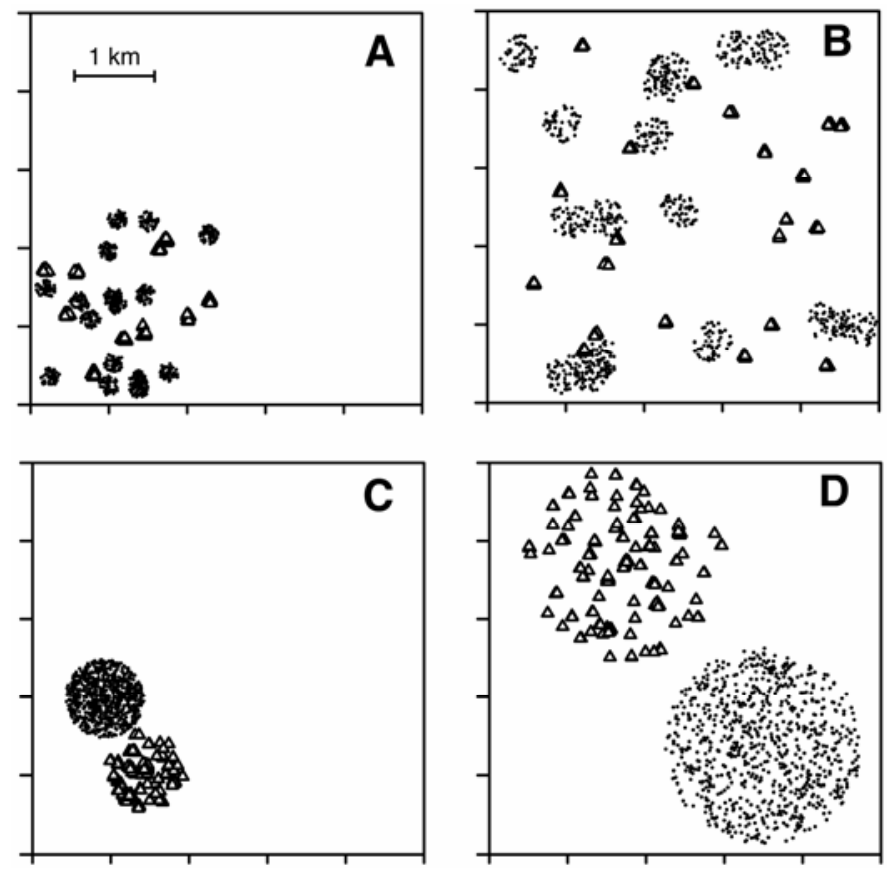

Fig. 1. The spatial arrangement of the four artificial host distributions containing residential $(\bullet)$ and commercial $(\triangle)$ citrus hosts. A, High-density interspersed with 15 clusters of residential hosts and 15 commercial hosts each of a radius of $150 \mathrm{~m}$. B, Low-density interspersed with 15 clusters of residential hosts and 15 commercial hosts each of a radius of $250 \mathrm{~m}$. C, Highdensity segregated with a single cluster of residential hosts and a single cluster of citrus hosts each of a radius of $500 \mathrm{~m}$. D, Low-density segregated with a single cluster of residential hosts and a single cluster of citrus hosts each of a radius of 1,250 $\mathrm{m}$. Each host distribution consists of 800 residential host units and 200 commercial host units. Individual host units are not drawn to scale. 
disease status (healthy or infected) of each host unit explicitly tracked. A description of all epidemiological parameters and their respective values is given in Table 1. Each timestep the probability that a healthy host unit $i$ of type $k$ becomes infected by inoculum from any existing infected host unit $j$ is given by

$$
\text { Prob } i \text { infected in } \Delta t=1-\exp \left[-\lambda_{k} N_{k}\left(\sum_{j \in \inf } \beta_{k} \phi_{j k} N_{k} \exp ^{-\alpha d_{i j}}\right)\right]
$$

where the contribution of inoculum from an infected host unit $j$ to a healthy host unit $i$ decreases exponentially with Euclidean distance $d_{i j}$ with exponent $\alpha$. Cook et al. (6) tested the negative power-law model and the Cauchy model but found the exponential provided the best fit to the data. For commercial host units we assume that both susceptibility and transmissibility scale linearly with the parameter estimates of Cook et al. (6) in proportion to their size. $\lambda_{k}$ represents the susceptibility of a tree of host type $k$ which is multiplied by the number of trees within the host unit (i.e., a single tree for a residential host and 100 trees for a commercial host) to give the total susceptibility of that host unit. Similarly, the transmission rate of inoculum, $\beta_{k}$, is multiplied by the number of infected trees at each host unit, $\phi_{j k} N_{k}$, where $\phi_{j k}$ is the fraction of trees infected at $j$ and $N_{k}$ is the total number of trees in the host unit. For residential host units $\sigma_{k}=N_{k}=1$ and $\phi_{j k} N_{k}=1$, which ensures the model collapses to that of the one host-type model of Cook et al. (6) for host distributions consisting of only residential trees. Infection within the commercial host units was represented by considering the fraction of infected trees, $\phi_{j k}$, as a variable that increases according to a deterministic susceptible-infected equation subsequent to initial infection

$$
\phi_{j k}(t-\tilde{t})= \begin{cases}1 & \text { if } k=\text { dooryard } \\ \frac{1}{1+\frac{1-\phi_{0 j k}(t)}{\phi_{0 j k}(t)} \exp (-r t)} & \text { if } k=\text { commercial }\end{cases}
$$

where $r$ is chosen such that a commercial block becomes approximately $100 \%$ infected (i.e., $\phi_{j k} \approx 1$ ) 100 days following the time of initial infection, $\tilde{t}$, which is representative of observations in the field (30). Furthermore, we assume that each commercial tree within a commercial host unit is twice as infectious as a residential tree due to the more pronounced flush and juvenile tissue that well maintained trees in commercial groves have in comparison with poorly managed and unmaintained residential trees (T. R. Gottwald, personal communication). We also assume that each commercial tree is less susceptible than a residential tree due to preventative management measures, such as the use of copper sprays, which are applied in cultivated plantations but would be highly unusual in privately owned residential trees (16). For example, Behlau et al. (2) showed in field trials in Brazil that the combined use of copper sprays and windbreaks reduced ACC incidence on leaves by at least 50\% compared with that in untreated plots.

\section{TABLE 1. Epidemic model parameters and values}

\begin{tabular}{llc}
\hline Symbol & Description & Value \\
\hline$N_{k}$ & $\begin{array}{l}\text { Number of trees at host type } k \text { (residential, } \\
\text { commercial) }\end{array}$ & $(1,100)$ \\
$\sigma_{k}$ & $\begin{array}{c}\text { Susceptibility of tree type } k \text { (residential, } \\
\text { commercial) }\end{array}$ & $(1,0.5)$ \\
$\beta_{k}$ & $\begin{array}{c}\text { Transmissibility of tree type } k \text { (residential, } \\
\text { commercial) }\end{array}$ & $\left(0.01^{\mathrm{a}}, 0.015\right)$ \\
$d_{i j}$ & $\begin{array}{c}\text { Euclidean distance in meters between suscepti- } \\
\text { ble host unit } i \text { and infected host unit } j\end{array}$ & Variable \\
$\alpha$ & $\begin{array}{c}\text { Exponent of negative exponential } \\
\text { dispersal function }\end{array}$ & $0.027^{\mathrm{a}}$ \\
$\phi_{j k}$ & $\begin{array}{c}\text { Fraction of host unit infected per time unit } \\
r\end{array}$ & Rate of increase in fraction of host unit infected \\
\hline
\end{tabular}

${ }^{\text {a }}$ Cook et al. (6).
Simulating the eradication process. The simulated eradication process is designed to mimic the process of eradication carried out by the joint USDA, APHIS/Florida Department of Agriculture and Consumer Services Citrus Canker Eradication Program (CCEP) in Florida between 1995 and 2005 (13). Each infected host exhibits detectable symptoms following a period of approximately 100 days, i.e., the number of days following infection when ACC symptoms are known to be best visualized on live trees (15). Consequently, this is represented by a Weibull distribution with mean and variance determined by data (15) and observations from the field (T. R. Gottwald, personal communication). The Weibull distribution is used in place of the normal due to its statistical flexibility and computational convenience in generating deviates by inversion. Further, unlike the normal distribution, the Weibull does not generate negative values and so is more realistic when describing events in time. The host distribution is assessed for disease every 30 days during which symptomatic host units are removed in addition to all other hosts within a predetermined 'control radius'. Continuous surveillance for ACC is common practice in areas where there is a high risk of ACC invasion. To simulate this, the survey is initiated from a randomly selected starting point within the first 30 days of the epidemic (i.e., the length of the survey interval). This process is continued until all infected trees (symptomatic, and infected but non-symptomatic) have been removed and the disease is eradicated from the host distribution. At the end of each simulation the total number of hosts removed from each host population (residential and commercial) is recorded and interpreted as the cost of eradication.

\section{RESULTS}

The common route of entry for invading ACC epidemics is via the international movement of infected material by the general population. This is considered to have been the case with the most recent ACC epidemic in Florida where the disease was first discovered in a residential area near Miami International Airport (27). Therefore, we focus on the scenario whereby an epidemic of the disease is initiated in the population of residential hosts and can subsequently spread to the commercial host population. Consequently, each run the epidemic is initiated in a random host within the residential population. For each host distribution we first describe the results generated in the absence of eradication (i.e., no host removals). Secondly, we describe the results pertaining to an eradication strategy and its impact on the epidemic and the host population.

Epidemic behavior in the absence of eradication. We describe the characteristics of the epidemic in the absence of eradication by its disease progress curve (Fig. 2) and by the 'transit incidence' in each of the host distributions (Fig. 3). The transit incidence is the proportion of residential hosts infected at the time of the first transition of the epidemic to the commercial population.

As expected, disease progress occurred at a higher rate in the high-density host distributions (Fig. 2A and C) compared with the low-density distributions (Fig. 2B and D). Additionally, disease progress was greater in the commercial population than the residential population in both the high-density host distributions (Fig. 2A and C). Disease progress was lowest in the commercial population in the segregated low-density host distribution (Fig. 2D), this was despite the initialization of the epidemic in the residential population and the wider range of incidences at which the epidemic made transit to the commercial population in the segregated case (Fig. 3C) compared with the interspersed case (Fig. 3A).

In the low-density host distributions (Fig. 1B and D) disease progress was similar in the residential population in both interspersed (Fig. 2B) and segregated distributions (Fig. 2D). However, disease progress was higher in the commercial population in 
the interspersed (Fig. 2B) compared with the segregated distribution (Fig. 2D). This was supported by the transit incidences which were lower in the interspersed (Fig. 3C) than the segregated distribution (Fig. 3D). Although, as described in the previous paragraph, this difference in transit incidence between the interspersed and segregated cases was also observed in the high-density host distributions (Fig. 3A and C) but without the same influence on relative disease progress between the two host types (Fig. 2A and C).

The effect of eradication. Here we consider the effect of eradication on the host population by considering the proportion
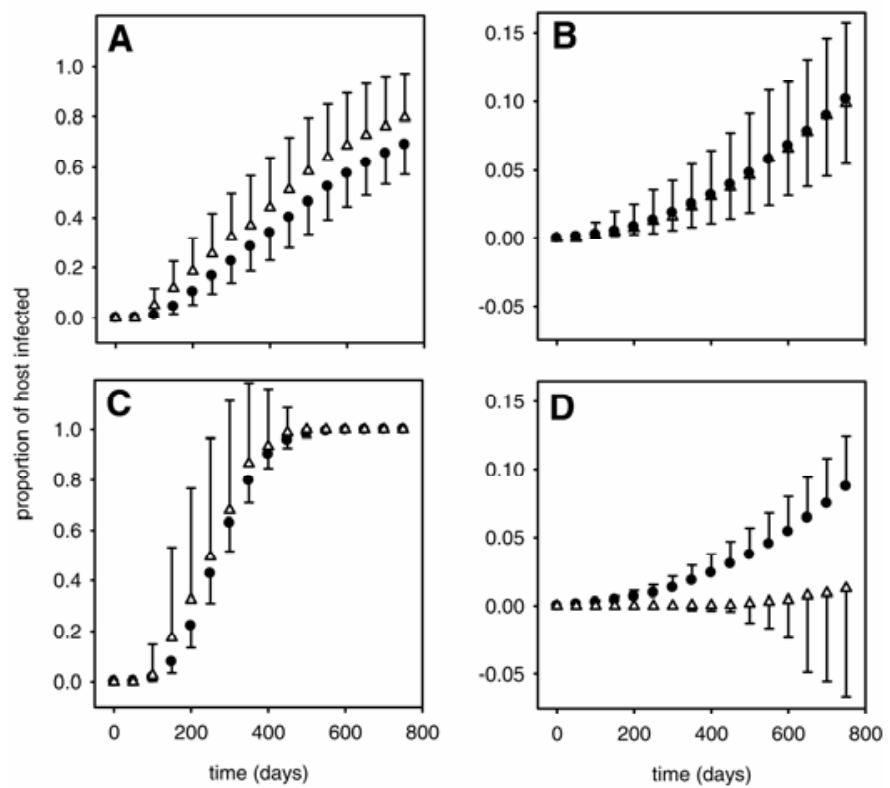

Fig. 2. Changes with time in the mean proportion of residential $(\bullet)$ and commercial $(\triangle)$ hosts infected in absence of eradication $(1,000$ replicates of the epidemic simulation model with unidirectional error bars shown [standarderror]). The figure labels correspond to each of the host distributions in Figure 1.
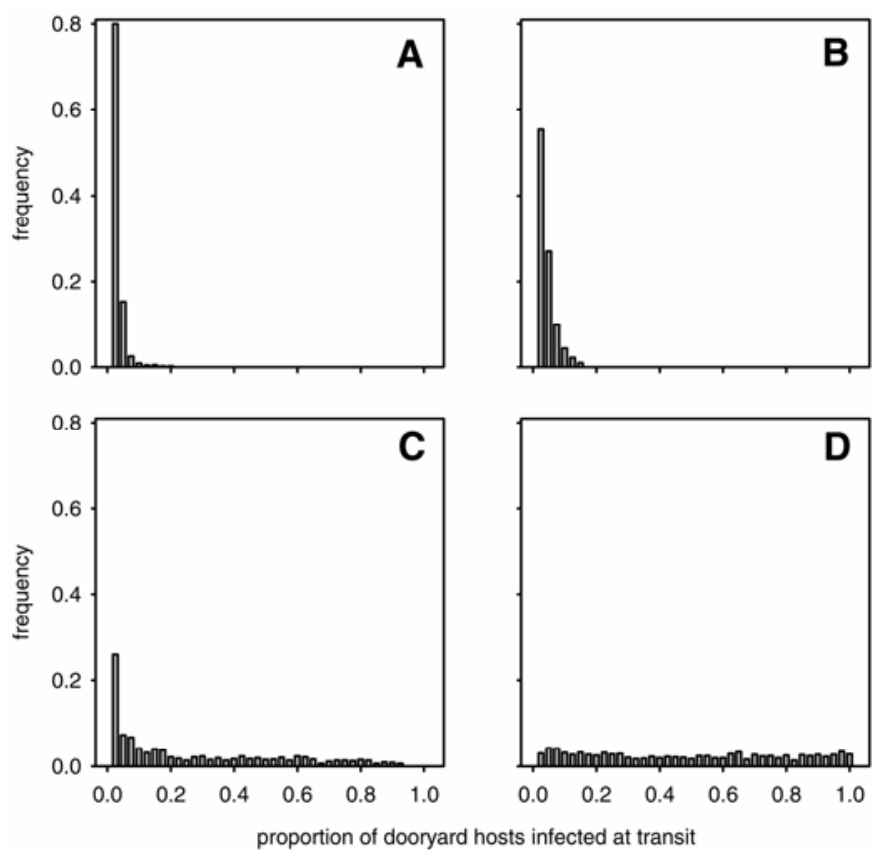

Fig. 3. Frequency distributions for the proportion of residential hosts infected at the time of the first transit of the disease to the commercial population in the absence of eradication (1,000 replicates of the epidemic simulation model). The figure labels correspond to each of the host distributions in Figure 1. of trees removed during the eradication of the disease (Fig. 4). The residual proportion of this is the proportion of healthy hosts remaining following eradication of the disease. We also consider the impact of the eradication program on the epidemic itself by recording the duration of the epidemic, i.e., the time to eradication (Fig. 5). In each case we look at the effect of varying the control radius, i.e., the radius around a detected symptomatic tree in which all neighboring trees (infected or not) are removed.

Two important results emerged. Firstly, an optimum control radius (i.e., a control radius which led to a minimum in the proportion of hosts removed) could be identified in both the resi-
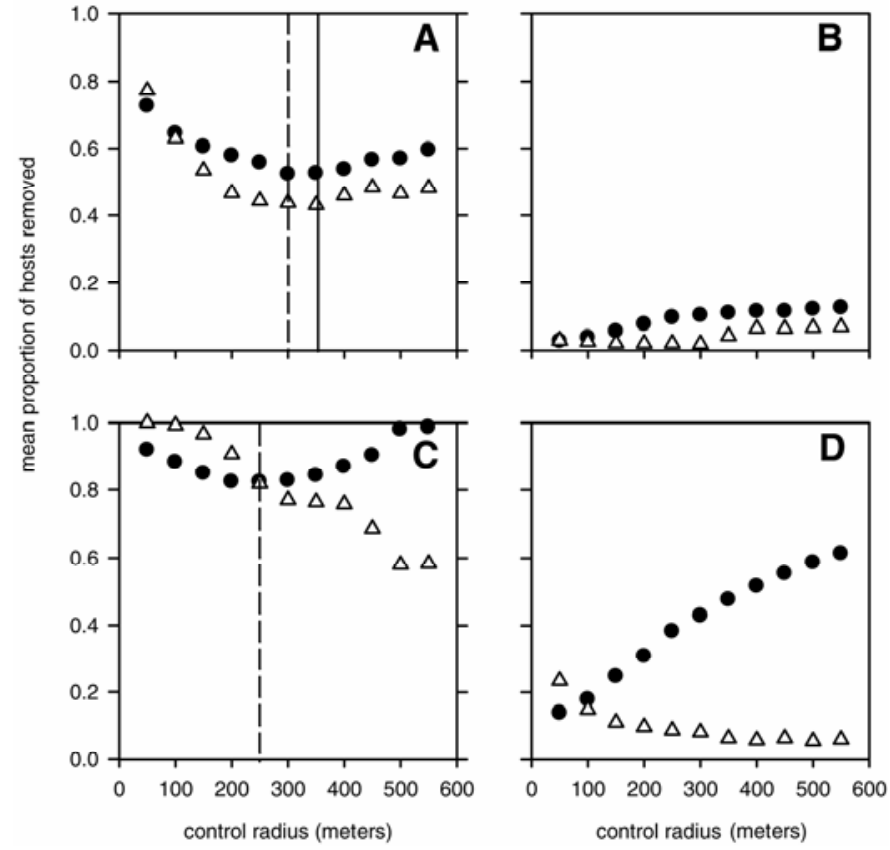

Fig. 4. The proportion of residential $(\bullet)$ and commercial $(\triangle)$ host units removed during eradication for varying lengths of control radius (mean of 1,000 replicates of the simulation model). The figure labels correspond to each of the host distributions in Figure 1.
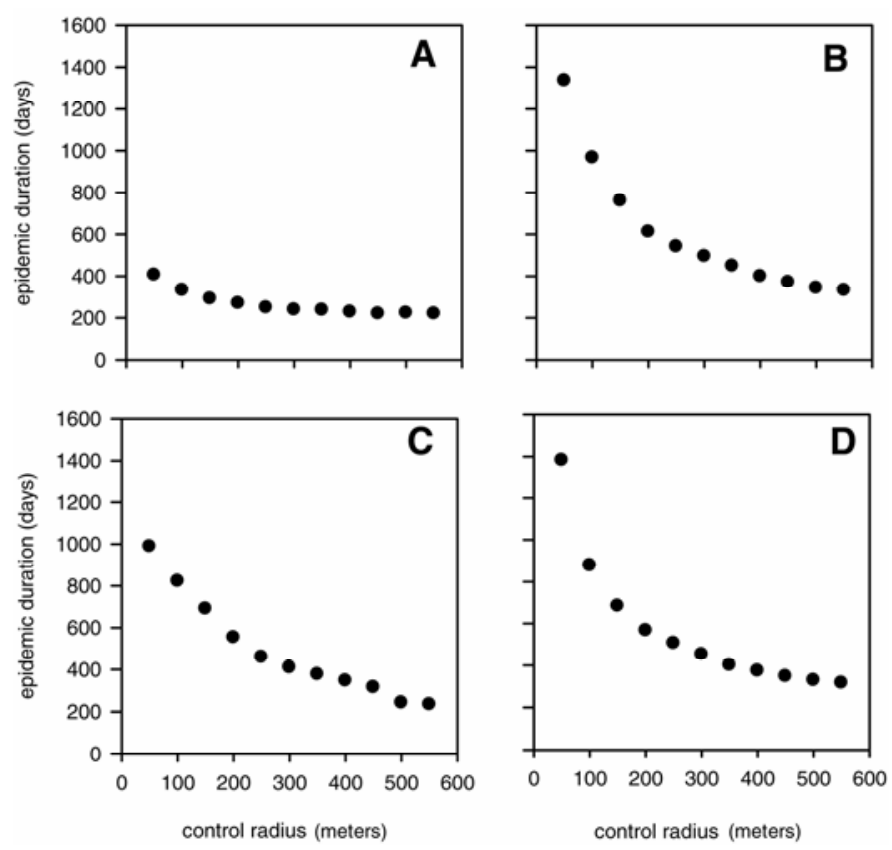

Fig. 5. The duration of an epidemic before eradication for varying lengths of control radius (mean of 1,000 replicates of the simulation model). The figure labels correspond to each of the host distributions in Figure 1. 
dential and commercial populations in the high-density interspersed host distribution (Fig. 4A). That is, increases in the control radius from 50 to $300 \mathrm{~m}$ and $350 \mathrm{~m}$ led to fewer host removals in the residential and commercial populations, respectively (Fig. 4A). However, increases in the control radius beyond this led to more removals in each host population (Fig. 4A). Secondly, in contrast with this, no optimum could be determined in the low-density segregated distribution (Fig. 4D) in either residential or commercial host population. Instead increases in the control radius led to increases in the proportion of residential hosts removed but decreases in the proportion of commercial hosts removed (Fig. 4D). The high-density segregated host distribution (Fig. 4C) showed a combination of these two contrasting results. An optimum control radius could be determined in the residential host population but any increase in the control radius decreased the proportion of hosts removed in the commercial population (Fig. 4C). The low-density interspersed distribution was associated with the fewest host removals in both the residential and commercial populations (Fig. 4B).

The duration of the epidemic generally decreased with increasing control radius in each of the host distributions (Fig. 5A to D) but was shortest in the high-density interspersed host distribution (Fig. 5A). There was no clear relationship between the proportion of hosts removed and the duration of the epidemic. For example, the proportion of total hosts removed was lowest in the lowdensity interspersed distribution (Fig. 4B); however, the epidemic duration was shorter in the high-density host distributions (Fig. 5A and $\mathrm{C}$ ) but longer in the low-density segregated distribution (Fig. 5D).

\section{DISCUSSION}

Any form of disease control involves costs, such as the cost of purchasing and applying a pesticide. When eradication is the goal and host removals are involved, this cost can be substantial. This is exemplified by the recent ACC eradication attempt in Florida which incurred a cost of $>\$ 1$ billion; a substantial component of which consisted of compensation payments to residential and commercial growers for trees removed by the eradication program (17). The calculation of optimal strategies which minimize such costs is vital to ensuring the feasibility of eradication programs. This problem has been the focus of recent human and animal epidemiological modeling studies which have determined optimal strategies in specific host populations given different levels of resource availability $(21,29)$. Here we show how the optimum strategy can differ for different host topologies and also the importance of delineating different host subgroups. In particular, we have shown that for ACC epidemics, depending on the host topology, eradication strategies can either minimize host removals in both residential and commercial populations (Fig. 4A) or minimize host removals in one host type at the expense of the other (Fig. 4D). Some care should be taken in making direct quantitative use of the results from this work as our study is restricted to epidemics occurring in relatively small and isolated host populations. In reality, epidemics of ACC will occur in much larger contiguous host populations and the epidemiological consequences of this will influence the scale of the control radius necessary to achieve eradication. However, we have made substantial progress in understanding how optimal strategies can differ for different host distributions. The key results from our study are summarized and discussed below.

Our results on the dynamics of the ACC epidemic in the various host distributions are comparable with ecological studies on the impact of habitat fragmentation on the population dynamics of different species $(19,20,28,31)$. The general result from this body of work is that "poor dispersers" can spread through fragmented landscapes if "stepping stones" of habitat exist between larger habitat patches. However, "good dispersers" remain relatively un- affected by landscape fragmentation and can move between habitat patches without the aid of stepping stones. In the current study, fragmentation is represented by the interspersed host distributions (Fig. 1A and B) and good and poor dispersal ability is analogous to varying the density of the host distribution (Fig. $1 \mathrm{C}$ and $\mathrm{D})$. The latter point is related to connectivity which can be enhanced by either decreasing the mean distance between host patches (i.e., density) or increasing the dispersal ability of the pathogen. In agreement with ecological studies, we find that in the low-density interspersed distribution incidence is enhanced in commercial hosts due to the increased proximity of commercial hosts to residential hosts which act as stepping stones linking the commercial population to the epidemic (Fig. 2B and D). However, in the high density host distributions, the incidence of the epidemic is higher in the segregated distribution than the interspersed. Here, the density is such that the stepping stone effect of the residential hosts is not necessary for early transmission to the commercial population and is in fact negated by the increased proximity of the highly transmissible commercial hosts to each other (Fig. 2).

We find that there is not a direct relationship between epidemic progress in the absence of eradication and the impact of eradication (in terms of epidemic duration and total number of host removed), i.e., an epidemic that is predicted to be larger in the absence of control is not necessarily harder to eradicate. The number of removals was lowest in both the high-density and lowdensity interspersed landscapes compared with their segregated counterparts despite the observation that disease progress was higher in the low-density interspersed distribution than the lowdensity segregated distribution. We conclude that the combination of the spatial removal of hosts and an existing spatially clumped host distribution (as exhibited by the interspersed host distributions [Fig. 1A and B]) acts to reduce the impact of eradication (in terms of both proportion of hosts removed and, in the highdensity case, the duration of the epidemic) via the spatial isolation of the epidemic. Similar conclusions have been found in studies of infectious diseases of animals. For example, using a spatially explicit simulation model, Eisinger and Thulke (8) found that spatially clustered fox populations required a lower immunization level for the eradication of rabies than nonclustered populations.

We find that there is an optimum radius for removal of hosts in the neighborhood of symptomatic hosts but that this depends on the topology of the host distribution. The existence of optimum control radii has been demonstrated for infectious diseases of livestock $(22,29)$. The existence of an optimum radius can be explained as follows. For a short control radius, the advancing wave of nonsymptomatic infected trees remains beyond the scale of control. However, for a very long control radius, we overstep the wave of nonsymptomatic infected trees and remove more trees than necessary to achieve eradication. In between these two extremes we find the optimum control radius. Here, we contribute to this body of work and show that the optimum depends critically on the topology of the host distribution. Additionally, we show that when considering multiple host populations the overall optimum must be found by minimizing some function of the removals in the two host populations. For hosts that are interspersed the optimum radius is very similar for both residential and commercial populations (Fig. 4A). However, in the lowdensity segregated distributions the optimums are in direct conflict with each other, i.e., the radius which minimizes removals in one population can maximize removals in another population (Fig. 4D). This occurs because by increasing the control radius the chance of the epidemic making transit to the commercial population is reduced.

This presents a political dilemma for regulators in that a price must be assigned to both residential trees and commercial tress in order to determine the optimal course of action. Although there are many more commercial trees than residential in the current 
study, residential trees have a disproportionate cost politically because each is represented by a single owner and public vote. In contrast, thousands of commercial trees can be owned by a single individual and vote. Thus, this balance of costs will have a large influence on the choice of eradication radius and will represent a politically contentious issue. In Florida, this is especially the case given that the citrus host landscape is characterized by segregated areas of residential and commercial citrus where the majority of commercial citrus is inland of the urbanized coastline. This is analogous to the segregated host distributions presented in this study (Fig. 1C and D and Fig. 4C and D). We have shown that this type of distribution results in greater host removals overall and can place the aims of eradication between the two host types in direct conflict with each other. These factors may have contributed to the negative media coverage and legal objections raised in the recent Florida citrus canker eradication program (12). However, the Texas citrus host landscape is predominantly characterized by an interspersion of residential and commercial citrus which is analogous to the interspersed host distributions (Fig. 1A and B and Fig. 4A and B). ACC has not yet invaded Texas; however, in the event of a future outbreak, an eradication program may have less adverse impact in terms of total host removals and additionally be less politically contentious in that the objectives of the commercial and residential citrus tree owners will be aligned (i.e., the control radius which minimizes host removals in one will also minimize removals in the other).

By using a spatially explicit stochastic simulation model, we have been able to capture the spatiotemporal dynamics of an ACC epidemic and eradication program that could not be achieved using nonspatial mean-field approaches more typical in the plant pathology literature. Crucially, we have shown that optimal strategies for ACC eradication can be identified but will depend on the topology of the host distribution. Moreover, by delineating between the two main categories of host, residential and commercial, we have highlighted that, in some circumstances, difficult judgments must be made between the relative cost of each host type before an overall optimal strategy can be identified. A final caveat to this work is that we consider an epidemic which is under surveillance for the disease every 30 days prior to epidemic initiation; therefore, early detection is assumed. Should an eradication program be initiated once the epidemic is at a high incidence (for example, if surveillance had not been in place prior to the initiation of the epidemic) or long breaks in eradication program beyond the standard 30-day interval occur, then this will greatly decrease the feasibility of eradication. This is exemplified by the recent Florida Citrus Canker Eradication Program where legal injunctions and consecutive hurricane spread events led to the abandonment of the eradication program when the disease was assessed to have spread too extensively for eradication to be achieved without removing an unfeasible number of host trees across the state (14). Finally, we must examine the lag in time between detection and tree removal, which in the present set of scenarios we held constant at 30 days. Physically, this lag is due to the logistics of moving manpower crews and machinery within the broad landscape combined with the legal requirement of obtaining permission to enter a private property for inspection, or court approval in the form of a warrant where permission is not forthcoming. In addition, as in Florida, legal precedent requires a set period of time to elapse for property owners to challenge the eradication/removal of trees that are diseased or within the prescribed eradication distance. In Florida, due to the necessity of the eradication program to deal with the logistics and obligation to comply with legal precedent, the effective detection-removal temporal lag was usually 60 to 120 days. Such adjustments or variability in temporal lag would be common to many eradication programs, irrespective of the particular disease. This delay in removal of infected trees increases the number of potential dis- persal events, the dispersal kernel, and the transit time, and thus effects and elongates the time required to achieve eradication and also the length of the control radius required. Exploration of the effect of variability in the detection-removal temporal lag is beyond aspects explored in the current study but will be the subject for future extensions of this work.

\section{ACKNOWLEDGMENTS}

S. Parnell was funded by the Biotechnology and Biological Sciences Research Council (BBSRC) and the United States Department of Agriculture. Rothamsted Research receives support from the BBSRC. C. A. Gilligan gratefully acknowledges the support of a BBSRC professorial fellowship.

\section{LITERATURE CITED}

1. Allen, L. J. S. 2003. An Introduction to Stochastic Processes with Applications to Biology. Pearson Prentice Hall, New Jersey.

2. Behlau, F., Belasque, J., Bergamin, A., Graham, J. H., Leite, R. P., and Gottwald, T. R. 2008. Copper sprays and windbreaks for control of citrus canker on young orange trees in southern Brazil. Crop Prot. 27:807-813.

3. Bock, C. H., Parker, P. E., and Gottwald, T. R. 2005. Effect of simulated wind-driven rain on duration and distance of dispersal of Xanthomonas axonopodis pv. citri from canker-infected citrus trees. Plant Dis. 89:7180

4. Brown, K. 2001. Florida fights to stop citrus canker. Science 292:22752276.

5. Condeso, T. E., and Meentemeyer, R. K. 2007. Effects of landscape heterogeneity on the emerging forest disease sudden oak death. J. Ecol. 95:364-375.

6. Cook, A. R., Gibson, G. J., Gottwald, T. R., and Gilligan, C. A. Constructing the effect of alternative intervention strategies on historic epidemics. J. Roy. Soc. Interface 5:1203-1213.

7. Diekmann, O., Heesterbeek, J. A. P., and Metz, J. A. J. 1990. On the definition and the computation of the basic reproduction ratio $R_{0}$ in models for infectious-diseases in heterogeneous populations. J. Math. Biol. 28:365-382.

8. Eisinger, D., and Thulke, H. H. 2008. Spatial pattern formation facilitates eradication of infectious diseases. J. Appl. Ecol. 45:415-423.

9. Garrett, K. A., Hulbert, S. H., Leach, J. E., and Travers, S. E. 2006. Ecological genomics and epidemiology. Eur. J. Plant Pathol. 115:35-51.

10. Gilligan, C. A., Truscott, J. E., and Stacey, A. J. 2007. Impact of scale on the effectiveness of disease control strategies for epidemics with cryptic infection in a dynamical landscape: An example for a crop disease. J. Roy. Soc. Interface 4:925-934.

11. Gottwald, T. R., Graham, J. H., Civerolo, E. L., Barrett, H. C., and Hearn, C. J. 1993. Differential host-range reaction of citrus and citrus relatives to citrus canker and citrus bacterial spot determined by leaf mesophyll susceptibility. Plant Dis. 77:1004-1009.

12. Gottwald, T. R., Graham, J. H., and Schubert, T. S. 2002. Citrus canker: The pathogen and its impact. Plant Health Progress doi:10.1094/PHP2002-0812-01-RV

13. Gottwald, T. R., Hughes, G., Graham, J. H., Sun, X., and Riley, T. 2001. The citrus canker epidemic in Florida: The scientific basis of regulatory eradication policy for an invasive species. Phytopathology 91:30-34.

14. Gottwald, T. R., and Irey, M. 2007. Post-hurricane analysis of citrus canker II: Predictive model estimation of disease spread and area potentially impacted by various eradication protocols following catastrophic weather events. Plant Health Progress doi:10.1094/PHP2007-0405-01-RS.

15. Gottwald, T. R., Sun, X., Riley, T., Graham, J. H., Ferrandino, F., and Taylor, E. L. 2002. Geo-referenced spatiotemporal analysis of the urban citrus canker epidemic in Florida. Phytopathology 92:361-377.

16. Gottwald, T. R., and Timmer, L. W. 1995. The efficacy of windbreaks in reducing the spread of citrus canker caused by Xanthomonas campestris pv. citri. Trop. Agric. 72:194-201.

17. Graham, J. H., Gottwald, T. R., Cubero, J., and Achor, D. S. 2004. Xanthomonas axonopodis pv. citri: Factors affecting successful eradication of citrus canker. Mol. Plant Pathol. 5:1-15.

18. Gubbins, S., and Webb, C. 2005. Simulation options for a national control programme to eradicate scrapie from Great Britain. Prev. Veterinary Med. 69:175-187.

19. Hale, M. L., Lurz, P. W. W., Shirley, M. D. F., Rushton, S., Fuller, R. M., and Wolff, K. 2001. Impact of landscape management on the genetic structure of red squirrel populations. Science 293:2246-2248.

20. Hanski, K., and Ovaskainen, O. 2003. Metapopulation theory for 
fragmented landscapes. Theor. Pop. Biol. 64:119-127.

21. House, T., and Keeling, M. J. 2008. Deterministic epidemic models with explicit household structure. Math. Biosci. 213:29-39.

22. Matthews, L., Haydon, D. T., Shaw, D. J., Chase-Topping, M. E., Keeling, M. J., and Woolhouse, M. E. J. 2003. Neighbourhood control policies and the spread of infectious diseases. Proc. Roy. Soc. Lond. Ser. B-Biol. Sci. 270:1659-1666.

23. May, R. M., and Anderson, R. M. 1988. The transmission dynamics of human immunodeficiency virus (HIV). Philosophical Trans. Roy. Soc. Lond. Ser. B-Biol. Sci. 321:565-607.

24. Neyman, J., and Scott, E. L. 1958. Statistical approach to problems in cosmology (with discussion). J. Roy. Stat. Soc. B 20:1-43.

25. Parnell, S., van den Bosch, F., and Gilligan, C. A. 2006. Large-scale fungicide spray heterogeneity and the regional spread of resistant pathogen strains. Phytopathology 96:549-555.

26. Plantegenest, M., Le May, C., and Fabre, F. 2007. Landscape epi- demiology of plant diseases. J. Roy. Soc. Interface 4:963-972.

27. Schubert, T. S., Rizvi, S. A., Sun, X., Gottwald, T. R., Graham, J. H., and Dixon, W. N. 2001. Meeting the challenge of eradicating citrus canker in Florida-Again. Plant Dis. 85:340-356.

28. Sondgerath, D., and Schroder, B. 2001. Population dynamics and habitat connectivity affecting the spatial spread of populations-A simulation study. Landsc. Ecol. 17:57-70.

29. Tildesley, M. J., Savill, N. J., Shaw, D. J., Deardon, R., Brooks, S. P., Woolhouse, M. E. J., Grenfell, B. T., and Keeling, M. J. 2006. Optimal reactive vaccination strategies for a foot-and-mouth outbreak in the UK. Nature 440:83-86.

30. Verniere, C. J., Gottwald, T. R., and Pruvost, O. 2003. Disease development and symptom expression of Xanthomonas axonopodis pv. citri in various citrus plant tissues. Phytopathology 93:832-843.

31. With, K. 2002. The landscape ecology of invasive spread. Conserv. Biol. 16:1192-1203. 